\title{
Changes in Cognitive Function after Left Atrial Appendage Occlusion
}

\section{Mona Laible ${ }^{*}$, Martin Andermann ${ }^{1}$, Claudia Jansen${ }^{1}$, Klaus Hess ${ }^{1}$, Nicolas Alexander Geis ${ }^{2}$, Sven Pleger ${ }^{2}$, Svenja Schüler ${ }^{3}$, Timolaos Rizos ${ }^{1}$, Roland Veltkamp ${ }^{1,4}$, and Solveig Horstmann ${ }^{1}$}

\author{
${ }^{1}$ Department of Neurology, Heidelberg University Hospital, Heidelberg, Germany \\ ${ }^{2}$ Department of Internal Medicine III, Heidelberg University Hospital, Heidelberg, Germany \\ ${ }^{3}$ Institute of Medical Biometry and Informatics, University of Heidelberg, Germany \\ ${ }^{4}$ Department of Stroke Medicine, Imperial College London, London, UK
}

*Corresponding author: Mona Laible, Department of Neurology, Heidelberg University Hospital, Heidelberg, Germany, Tel: +49 6221567504 ; Fax: +49 622156 5670; E-mail: mona.laible@med.uni-heidelberg.de

Rec Date: February 04, 2018; Acc Date: February 21, 2018; Pub Date: February 26, 2018

Citation: Laible M, Andermann M, Jansen C, Hess K, Geis NA, et al. (2018) Changes in Cognitive Function after Left Atrial Appendage Occlusion. J Neurol Neurosci Vol.9 No.1:248.

\section{Abstract}

Background: Cognitive dysfunction is a frequent phenomenon after surgical and cardiovascular interventions. No data on cognitive function after left atrial appendage occlusion (LAAO) are available so far. The aim of the present study was to evaluate the cognitive function after LAAO compared to before LAAO.

Methods and findings: Patients who underwent LAAO for treatment of atrial fibrillation at the Heidelberg Department of Internal Medicine III were eligible for this observational, explorative, single-center, non-randomized cohort study, between July 2013 and January 2016. Neurological examination and neuropsychological assessments were conducted one day before LAAO and one month after LAAO, using a comprehensive neuropsychological test battery that included several cognitive domains including executive function, verbal fluency, verbal and non-verbal memory. Paired t-tests and correlation analyses were applied to compare test results pre- and post-intervention. In addition, we descriptively analyzed the number of relevant changes ( \pm 1 standard deviation) over all cognitive domains for each patient. 20 patients completed the study. Mean age was $72.6 \pm 6.8$ years and 15 (75\%) were male. There were no significant changes in any of the tested cognitive domains in group statistics. Descriptive single case analysis showed more deteriorations than improvements by one standard deviation over all cognitive domains in three patients, while in 11 patients the number of positive changes exceeded the number of negative changes.

Conclusion: LAAO does not adversely affect cognitive function in the majority of cases.
Keywords: Atrial fibrillation; Cognitive function; Cognitive impairment; Left atrial appendage occlusion; Neuropsychology; Safety

\section{Abbreviations}

AF: Atrial Fibrillation; CERAD: Consortium to Establish a Registry for Alzheimer's Disease Neuropsychological Battery; CHA2DS2vasc Score: C=Congestive Heart Failure; $\mathrm{H}=$ Hypertension; $\mathrm{A2}=\mathrm{Age} \geq 75$ Years (Double Risk Weight); $\mathrm{D}=$ Diabetes Mellitus; $\mathrm{S}=$ Previous Stroke/TIA/Arterial Embolism (Double Risk Weight); V=Vascular Disease; A=Age 65-74 Years; $\mathrm{Sc}=($ Female) Sex Category which Gives a Total Score from 0 to 9 for Estimating the Risk of Stroke in Patients with NonRheumatic Atrial Fibrillation; CLOX: A Clock Drawing Task to Test Executive Function; DESC: Rasch-Based Depression Screening; HAS-BLED Score: An Abbreviation of "Hypertension, Abnormal Renal and Liver Function, Stroke, Bleeding, Labile INR, Elderly Age, Drugs or Alcohol", Composing a Score to Assess 1-Year Risk of Major Bleeding in Patients with Atrial Fibrillation; ICH: Intracerebral Hemorrhage; IQR: Interquartile Range; LAAO: Left Atrial Appendage Occlusion; Moca: Montreal Cognitive Assessment; MRS: Modified Rankin Scale; NIHSS: National Institutes of Health Stroke Scale; POCD:

Postoperative Cognitive Dysfunction; SD: Standard Deviation; TAVI: Transcatheter Aortic Valve Implantation; TIA: Transient Ischemic Attack; TMT-A/-B: Trail Making Test Versions A And B

\section{Introduction}

Postoperative cognitive dysfunction (POCD), defined as a new cognitive impairment arising after a surgical procedure [1], is a frequent postoperative phenomenon. However, data on its incidence are inconsistent and depend on the procedure. POCD was described in cardiac surgery as coronary artery bypass surgery $[2,3]$, but also in extracardiac surgery $[4,5]$. Elderly people and patients with coronary heart disease seem to be especially vulnerable for POCD, but as many as $36.6 \%$ of surgical patients aged between 18 and 39 years are 
also affected by POCD [4]. On the other hand, meta-analysis of 2043 patients receiving coronary artery bypass graft surgery suggested an improvement in cognitive function in the first year following coronary artery bypass surgery [2]. POCD is not restricted to open cardiac surgical procedures but has also been reported in less invasive procedures as cardiac interventional approaches including ablation [6] and transcatheter aortic valve implantation (TAVI) [7,8]. Overall, however, in TAVI, a review resumed that global cognition improved or remained unchanged [8].

Besides age and concomitant cardiovascular disease, the extent of the surgical procedure has been identified as a risk factor for the development of POCD [1]. In contrast, no clear association between POCD and general or regional types of anesthesia has been established so far $[1,9]$.

In patients with atrial fibrillation (AF), current US- and European guidelines consider left atrial appendage occlusion (LAAO) as an alternative for the prevention of stroke when anticoagulation is contraindicated $[10,11]$. Due to the high age and frequent concomitant cardiovascular diseases in patients eligible for $L A A O$, these patients could be at a relevant risk for POCD.

In this study, we aimed to explore the prevalence of cognitive deterioration one month after percutaneous LAAO compared to pre-procedural assessment.

\section{Methods}

This prospective consecutive observational cohort study was performed at the Heidelberg University Hospital after approval of the local independent ethics committee, and all investigations have been conducted according to the principles expressed in the Declaration of Helsinki of 1964 and its later amendments. Study procedures are summarized in Figure 1.

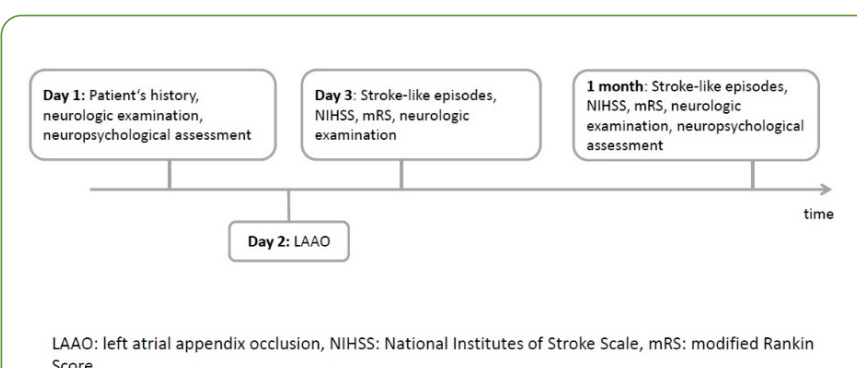

Figure 1 Summary of study procedures.

All patients who were planned to undergo LAAO between July 2013 and January 2016 were eligible. This study was part of a comprehensive project to describe the cognitive changes and the frequency of silent brain infarcts after LAAO [12]. Both studies had an overlap of patients. The inclusion criteria encompassed: (1) oral and written informed consent to take part in the study; (2) age $\geq 18$ years; and (3) sufficient skills of German language, as all neuropsychological exams were performed using German test versions. Exclusion criteria were:
(1) denial to participate in the study; (2) symptoms of aphasia, as identified with the 'Token Test' [13]; (3) visual neglect, as identified by the CATS test [14]; (4) symptoms of major depression, as revealed with the Rasch-based Depression Screening (DESC) questionnaire ([15]; cutoff value: sum score $\geq$ 12 points pre-LAAO or mean value of DESC pre-/post-LAAO $\geq$ 12 points). The primary study endpoint was cognitive function one month after LAAO. Secondary endpoints were postinterventional ischemic stroke, transient ischemic attack (TIA) and intracerebral hemorrhage (ICH) after LAAO. Diagnoses of TIA, ischemic stroke and ICH were made according to standard criteria [16-18]. We recorded clinical characteristics of all patients at baseline, including the type of AF (paroxysmal or permanent), the presence of arterial hypertension, diabetes, history of intra- or extracerebral hemorrhage, ischemic stroke or TIA.

Table 1 Patient characteristics.

\begin{tabular}{|c|c|}
\hline \multicolumn{2}{|l|}{ Patient characteristics } \\
\hline Age, mean (SD) & $72.6(8.5)$ \\
\hline Male sex, $\mathrm{n}(\%)$ & $15(75.0)$ \\
\hline Prior ischemic stroke, $\mathrm{n}(\%)$ & $4(20.0)$ \\
\hline Prior TIA, n (\%) & $3(15.0)$ \\
\hline Prior $\mathrm{ICH}, \mathrm{n}(\%)$ & $10(50.0)$ \\
\hline Prior systemic hemorrhage, $\mathrm{n}(\%)$ & $10(50.0)$ \\
\hline$A F$, paroxysmal n (\%) & $11(55.0)$ \\
\hline permanent & $9(45.0)$ \\
\hline Arterial Hypertension, $\mathrm{n}(\%)$ & $19(95.0)$ \\
\hline Diabetes, n (\%) & $6(30.0)$ \\
\hline Vascular disease, $\mathrm{n}(\%)$ & $12(60.0)$ \\
\hline CHA2DS2vasc score, mean (SD) & $4.5(1.7)$ \\
\hline HAS-BLED score, mean (SD) & $3.3(0.7)$ \\
\hline History of OAC, $n(\%)$ & $6(30.0)$ \\
\hline NIHSS pre-intervention, mean (SD) & $0.8(1.7)$ \\
\hline mRS pre-intervention, mean (SD) & $2.0(0.9)$ \\
\hline NIHSS post-intervention, mean (SD) & $0.8(1.7)$ \\
\hline mRS post-intervention, mean (SD) & $2.0(0.9)$ \\
\hline \multicolumn{2}{|l|}{ LAAO device, n (\%) } \\
\hline Watchman & $3(15.8)$ \\
\hline Amplatzer & $14(73.7)$ \\
\hline Amulet & $2(10.5)$ \\
\hline
\end{tabular}

Additionally, we determined the CHAD2DS2vasc and the HAS-BLED scores [19]. Within the neurological examination at day 1 (before LAAO) and day 2 as well as one month after LAAO, the National Institutes of Health Stroke Scale (NIHSS) and the modified Rankin Scale Score (mRS) were determined for each patient. 


\section{Patient's characteristics}

A flow-chart of the study population is provided in Figure 2. A total of 27 patients were enrolled. Of those, 7 patients had to be excluded from final analysis, of whom one male patient suffered a periinterventional stroke due to air embolism and subsequent inability to undergo neuropsychological assessment post-LAAO (Figure 2).

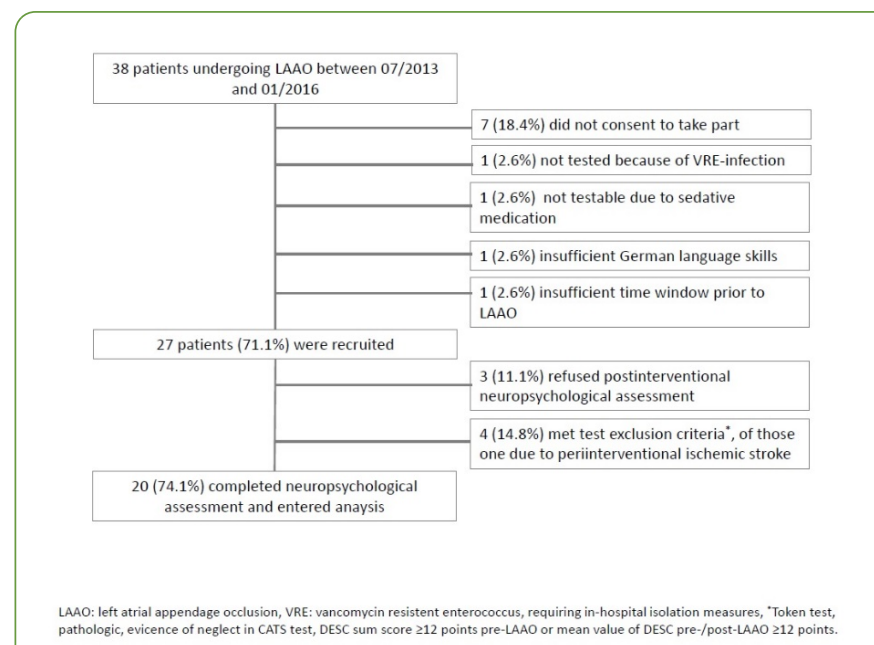

Figure 2 Flow-chart of the study population.

A total of 20 patients completed the study assessments. Mean age was $72.6 \pm 8.5$ years. 15 patients were male $(75.0 \%)$; 11 patients had paroxysmal (55.0\%) and 9 had persistent AF (45.0\%). Patient characteristics are summarized in Table 1.

\section{Neuropsychological assessment}

Assessment pre- and post- LAAO comprised the following cognitive tests: Montreal Cognitive Assessment (MoCA) [20]; CLOX, a clock drawing task to test executive function [21]; the German version of Consortium to Establish a Registry for Alzheimer's Disease neuropsychological battery (CERAD-plus) $[22,23]$, including the subtests semantic and phonemic verbal fluency, Boston naming test, verbal memory, non-verbal memory, and visuo-spatial abilities and trail making test (versions $A$ and $B$, TMT-A/-B) [24]; digit span (forward and backward). All neuropsychological assessments were conducted by two clinical neuropsychologists $(\mathrm{CJ}, \mathrm{KH})$ and a fully-trained psychologist (MA).

\section{Left atrial appendage occlusion}

LAAO was performed by two experienced interventional cardiologists (NG, SP) according to local routine practice. The study had no influence on the procedure. An individualized anesthetic approach was used with local inguinal and general anesthesia using midazolam and/or propofol, respectively. Additional administration of intravenous morphine was possible. Routine measures of oxygenation, blood pressure and heart rate monitoring were documented. LAAO guidance was assured by transesophageal echocardiography combined with fluoroscopy. Access to the left atrium was obtained via the right femoral vein and subsequent transseptal puncture. The occluder (Amplatzer Cardiac Plug and Amulet device, both St. Jude Medical, Saint Paul, Minnesota, USA; or WATCHMAN ${ }^{\mathrm{TM}}$ device, Boston Scientific, Marlborough, Massachusetts, USA) was implanted into the left atrial appendage. After the procedure, antithrombotic treatment was started with aspirin and clopidogrel for the first three months, followed by a monotherapy with aspirin.

\section{Follow-up}

Follow-up was conducted one month after LAAO during a personal visit. The study follow-up included the patient's interim history for ischemic and hemorrhagic stroke, TIA, systemic emboli, intra- or extracranial bleeding events, a neurological examination including the NIHSS and the mRS, and the repetition of the neuropsychological assessment.

\section{Statistical analysis}

Statistical analyses were performed using the Statistical Package for the Social Sciences (version 22.0; SPSS Inc., Chicago, Illinois, USA) and MATLAB (Version 9.0; The MathWorks, Inc., Natick, Massachusetts, USA). Continuous data were checked for normal distribution using the Kolmogorov-Smirnov test. Accordingly, in the remainder of this paper; they are reported as means and standard deviations (SD) or medians and interquartile ranges (IQR). Categorical data are presented as frequencies and percentages. Measured values pre- and post-LAAO were evaluated using paired Student's t-test and Pearson correlation for normally distributed data and using Wilcoxon's signed-rank test and Spearman correlation in the case of not normally distributed data. A p-value $<0.05$ was considered statistically significant. Whenever an individual patient showed a change of a cognitive test result in post-LAAO testing exceeding \pm 1 SD compared to pre-LAAO test results, this was regarded as a deterioration or improvement in the respective test [25]. Since changes of cognitive scores may occur quite frequently within a battery of 13 test variables, we offset all improvements against all deteriorations in each patient and considered only a net change of $\geq 1$ in either direction as relevant.

\section{Results}

\section{Clinical neurological assessment}

NIHSS (mean $0.8 \pm 1.7$ ) and mRS (mean $2.0 \pm 0.86$ ) were unchanged in the study sample pre- and post-intervention. At one month, none of the patients had suffered a TIA, an ischemic stroke or a clinically evident $\mathrm{ICH}$. All patients were treated with dual antiplatelet therapy with ASS and clopidogrel at the follow-up visit at one month.

\section{Neuropsychological assessment- group analysis}

Table 2 displays the results of the statistical evaluations for each of the neuropsychological tests. When comparing pre- 
and post-interventional assessments, there occurred no significant change in any of the tests; moreover, the correlations between pre- and post-LAAO test results were significantly positive in any of the subtests.

Table 2 Results of Neuropsychological assessment.

\begin{tabular}{|c|c|c|c|c|c|c|}
\hline \multirow[t]{2}{*}{ Variables } & \multirow{2}{*}{$\begin{array}{l}\text { pre-LAAO } \\
\text { mean } \pm \text { SD }\end{array}$} & \multirow{2}{*}{$\begin{array}{l}\text { post-LAAO } \\
\text { mean } \pm \text { SD }\end{array}$} & \multicolumn{2}{|c|}{ Pearson correlation } & \multicolumn{2}{|c|}{ paired t-test } \\
\hline & & & $\mathbf{r}^{2}$ & $\mathbf{p}$ & $\mathbf{t}$ & $\mathbf{p}$ \\
\hline MoCA & $24.1 \pm 2.2$ & $24.0 \pm 3.4$ & 0.76 & $<0.001^{* * *}$ & 0.1 & 0.92 \\
\hline \multicolumn{7}{|c|}{ CLOX } \\
\hline part 1 & $12.1 \pm 2.3$ & $11.7 \pm 2.7$ & 0.69 & $0.001^{\star *}$ & 0.89 & 0.39 \\
\hline part 2 & $13.2 \pm 1.8$ & $13.6 \pm 1.4$ & 0.66 & $0.002^{* *}$ & -1.32 & 0.2 \\
\hline \multicolumn{7}{|c|}{ TMT } \\
\hline part A & $48.0 \pm 15.1$ & $47.6 \pm 14.6$ & 0.78 & $<0.001^{* * *}$ & 1.03 & 0.32 \\
\hline part B & $133.2 \pm 73.5$ & $119.2 \pm 54.1$ & 0.8 & $<0.001^{\star * *}$ & 1.98 & 0.06 \\
\hline Boston Naming Test & $14.2 \pm 1.1$ & $14.4 \pm 1.0$ & 0.61 & $0.006^{* *}$ & -0.72 & 0.48 \\
\hline \multicolumn{7}{|c|}{ CERAD verbal fluency } \\
\hline Animals & $18.9 \pm 5.0$ & $19.8 \pm 5.8$ & 0.76 & $<0.001^{\star \star \star}$ & -1.11 & 0.28 \\
\hline s-words & $8.8 \pm 5.6$ & $9.5 \pm 3.6$ & 0.48 & $0.033^{*}$ & -0.79 & 0.44 \\
\hline CERAD verbal recall & $5.2 \pm 1.6$ & $6.1 \pm 2.0$ & 0.53 & $0.016^{*}$ & 0.96 & $0.05^{*}$ \\
\hline \multicolumn{7}{|c|}{ Digit span } \\
\hline Forward & $5.7 \pm 0.9$ & $5.7 \pm 0.9$ & 0.63 & $0.003^{* *}$ & $<0.01$ & 1 \\
\hline Backward & $4.0 \pm 0.8$ & $3.8 \pm 1.3$ & 0.67 & $0.001^{* *}$ & 0.94 & 0.36 \\
\hline \multirow[t]{2}{*}{-} & pre-LAAO & post-LAAO & \multicolumn{2}{|c|}{ Spearman correlation } & \multicolumn{2}{|c|}{ Wilcoxon test } \\
\hline & median (IQR) & median (IQR) & r2 & $\mathbf{P}$ & $\mathbf{z}$ & $\mathbf{p}$ \\
\hline CERAD constructive praxis & $10.2(10-11)$ & $11.0(10-11)$ & 0.74 & $<0.001^{* \star *}$ & -1.41 & 0.16 \\
\hline CERAD figural recall & $7.0(5-11)$ & $9.0(7-11)$ & 0.6 & $0.005^{* *}$ & -1.84 & 0.07 \\
\hline
\end{tabular}

A post-interventional improvement in the CERAD-plus verbal recall test showed a trend towards significance $(p=0.05)$; similarly, there appeared non-significant trends for improvement in the TMT-B $(p=0.06)$, as well as in the CERADplus figural recall $(p=0.07)$.

\section{Neuropsychological assessment - single case analysis}

Figure 3 depicts all individual test results pre- (T1) and post(T2) LAAO, separately for each component of the neuropsychological examination; each participant is assigned an individual upper-case letter. In some participants, the individual scores of a given test deteriorated pre-/post-LAAO by $>1 \mathrm{SD}$; the respective individuals are denoted in black letters in Figure 3, and the corresponding shift is illustrated using bold black lines. With the exception of TMT A/B and CERAD-plus constructive praxis, at least one such instance occurred in any of the conducted tests.

The summary plot in the lower part of Figure $\mathbf{3}$ shows the number of improvements and deteriorations for each patient. Three patients showed a greater number of deteriorations in test scores, while in 11 patients the number of positive changes exceeded the number of negative changes.

\section{Discussion}

There were no significant changes of the cognitive performance within the group analysis with a non-significant trend towards an improvement in three variables. Our descriptive single case analysis showed a net improvement, defined by the number of positive changes minus the number of negative changes over all test variables, in 11 patients, while three patients had a net deterioration. Of these three patients, one had a decline $\geq 1$ SD in three of the test variables, one other patient in two tests, and both without an improvement in any of the other tests. According to typical criteria in the POCD literature this could be interpreted as a cognitive decline [25]. However, since this study did not enroll a control group, the base rate of such changes is unknown; and given the large number of cognitive variables we employed, it cannot be ruled out that they did occur by chance alone. 


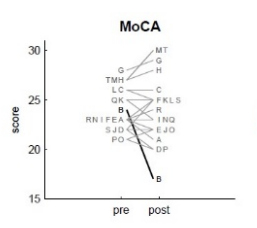

TMT A

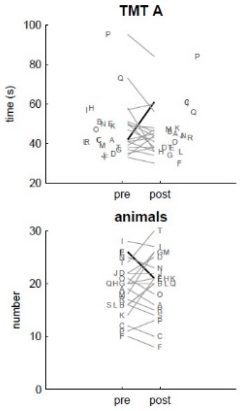

forward span
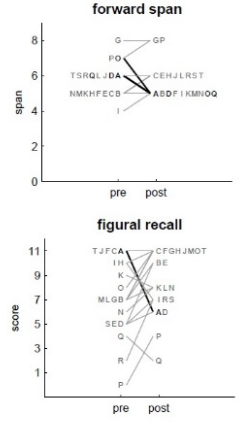

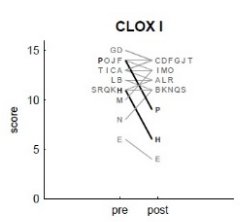

TMT B

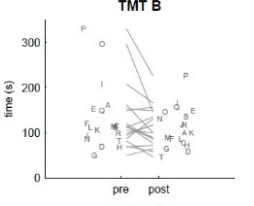

s-words
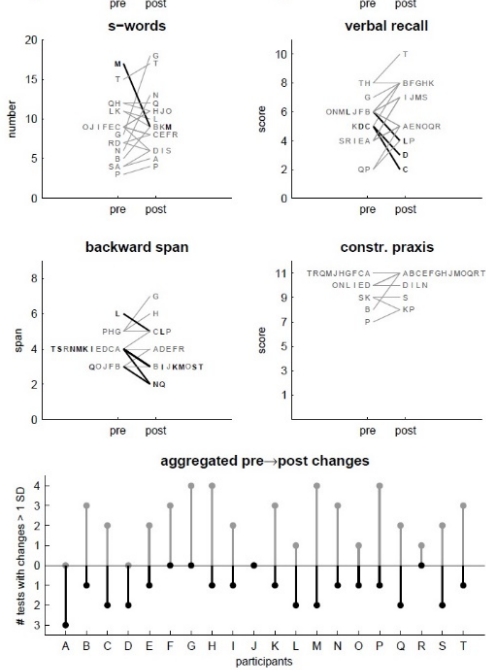

Figure 3 Course of test results on individual patient level pre- and post-LAAO. Any deterioration of $>1$ SD in test results is indicated by bold lines. T1: pre-left atrial appendage occlusion (LAAO), T2: post-LAAO. Patients No. 1-20 are indicated here as single letters (A-T) to allow individual assignment of test results; patients' letters are printed bold when a deterioration of test results $\geq 1$ SD was present. The summary panel in the lower part of the figure illustrates the number of post-LAAO tests in which the individual participants improved (grey) or deteriorated (black) by $\geq 1$ SD.

Our group findings are consistent with a previous study analyzing cognitive function after left atrial catheter ablation for AF in which no cognitive deficit was found on detailed neuropsychological assessment within 6 months after ablation [26]. After pulmonary vein isolation in AF, no clinically overt cognitive deficit developed upon 6 months [27]. These results are consistent with those of a metanalysis on cognitive function after coronary artery bypass graft surgery [2]. Their medium term-results showed a post-operative decline in two of four main tests indicating a decrease in psychomotor speed within two weeks after coronary artery bypass graft surgery, but a significant improvement relative to baseline in all four measures by three months [2]. Thus, the time of postinterventional assessment is an important factor. To minimize the effect of immediate interventional procedures including anesthesia on cognitive performance, we performed the postinterventional neuropsychological exam one month after the procedure. However, a consensus on optimal timing to test for POCD has not been established [2]. Moreover, we used different test batteries compared to previous work [26] and tested more single domains than previous studies [27] trying to describe cognitive function in detail. Comparability of our findings is therefore limited as different times of postoperative testing were chosen and composition of neuropsychological assessment was different. A further impact on the patient's cognitive and motor function may consist in new postinterventional ischemic brain lesions. Silent ischemic brain lesions are detected in 75 to $90 \%$ of patients after TAVI [28] but occur only in $4.8 \%$ in LAAO patients [12]. However, a review of the cognitive performance after TAVI suggests a stable or improved global cognition 3 months postoperative [8]. Studies correlating diffusion weighted imaging (DWI)lesions and cognitive function in cardiovascular procedures have not established a relationship between peri-procedural cognitive dysfunction and silent DWI lesions [26,29]. Further studies with combined MRI- and longitudinal neuropsychological assessment might clarify the clinical significance of silent post-procedural DWI-lesion for cognitive function.

The strengths of our study include the comprehensive test battery applied by fully-trained psychologists and the prospective and consecutive enrollment. Limitations are the single center approach, the small sample size and the absence of a control group. We did not collect data on patient-related factors like preoperative cognitive impairment, habits and drug use. Furthermore, practice effects may have influenced our results. Such effects have been reported in both CERAD and TMT-B $[30,31]$.

\section{Conclusion}

To conclude based on our group level data, LAAO appears to have no negative cognitive sequelae in an elderly patient cohort with AF. To confirm this finding and to evaluate the rate of POCD in single cases a controlled trial with a larger and ideally multicenter patient cohort is necessary.

\section{Acknowledgments}

The authors would like to thank Johanna Mair-Walther, PhD, for help with the neuropsychological assessment.

\section{Competing Interests}

ML was supported by a research fellowship of the Medical Faculty, University of Heidelberg during the course of the study. NAG received congress attendance and lecture fees from W.L. Gore and Associates, Inc. and lecture fees from St. Jude Medical, Inc. TR received consulting honoraria, speakers' honoraria and travel support from BMS Pfizer, Boehringer Ingelheim, Bayer HealthCare and Daiichi Sankyo. RV received consulting honoraria, research support, travel grants, and speakers' honoraria from Bayer HealthCare, Boehringer Ingelheim, BMS Pfizer, Daiichi Sankyo, Roche Diagnostics, St. 
Jude Medical, and Sanofi Aventis. MA, CJ, KH, SP, SS and SH report no competing interests.

\section{References}

1. Rundshagen I (2014) Postoperative cognitive dysfunction. Dtsch Arztebl Int 111: 119-125.

2. Cormack F, Shipolini A, Awad WI, Richardson C, McCormack DJ, et al. (2012) A meta-analysis of cognitive outcome following coronary artery bypass graft surgery. Neurosci Biobehav Rev 9 : 2118-2129.

3. Kennedy ED, Choy KC, Alston RP, Chen S, Farhan-Alanie MM, et al. (2013) Cognitive outcome after on- and off-pump coronary artery bypass grafting surgery: a systematic review and metaanalysis. J Cardiothorac Vasc Anesth 27: 253-265.

4. Monk TG, Weldon BC, Garvan CW, Dede DE, van der Aa MT, et al. (2008) Predictors of cognitive dysfunction after major noncardiac surgery. Anesthesiology 108: 18-30.

5. Aceto P, Perilli V, Lai C, Ciocchetti P, Vitale F, et al. (2015) Postoperative cognitive dysfunction after liver transplantation. Gen Hosp Psychiatry 37: 109-115.

6. Medi C, Evered L, Silbert B, Teh A, Halloran K, et al. (2013) Subtle post-procedural cognitive dysfunction after atrial fibrillation ablation. J Am Coll Cardiol 62: 531-539.

7. Miller DC, Blackstone EH, Mack MJ, Svensson LG, Kodali SK, et al. (2012) PARTNER Trial Investigators and Patients; PARTNER Stroke Substudy Writing Group and Executive Committee. PARTNER Stroke Substudy Writing Group and Executive Committee. Transcatheter (TAVR) versus surgical (AVR) aortic valve replacement: occurrence, hazard, risk factors, and consequences of neurologic events in the PARTNER trial J Thorac Cardiovasc Surg 4: 832-843.

8. Lai KS, Herrmann N, Saleem M, Lanctôt KL (2015) Cognitive outcomes following transcatheter aortic valve implantation: A systematic review. Cardiovasc Psychiatry Neurol 2015: 209569.

9. Evered L, Scott DA, Silbert B, Maruff P (2011) Postoperative cognitive dysfunction is independent of type of surgery and anesthetic. Anesth Analg 5: 1179-1185.

10. January $\mathrm{CT}$, Wann LS, Alpert JS, Calkins $\mathrm{H}$, Cigarroa JE, et al. (2014) American College of Cardiology/American Heart Association Task Force on Practice Guidelines. AHA/ACC/HRS guideline for the management of patients with atrial fibrillation: a report of the American College of Cardiology/American Heart Association Task Force on Practice Guidelines and the Heart Rhythm Society. J Am Coll Cardiol 64: 2305-2307.

11. Camm AJ, Lip GY, De Caterina R, Savelieva I, Atar D, et al. (2012) 2012 focused update of the ESC Guidelines for the management of atrial fibrillation: an update of the 2010 ESC Guidelines for the management of atrial fibrillation: developed with the special contribution of the European Heart Rhythm Association. Eur Heart J 33: 2719-2747.

12. Laible M, Möhlenbruch M, Horstmann S, Pfaff J, Geis NA, et al. (2017) Silent cerebral infarcts after left atrial appendage occlusion. Eur J Neurol 24: 53-57.

13. Huber W, Poeck K, Weniger D, Willmes K. Aachener Aphasie Test. Göttingen: Hogrefe. 1983.

14. Haid T, Hoch-Städele M, Pech C, Kofler M, Saltuari L (2010) Normen für ein Screeningverfahren zur Neglectdiagnostik.
Poster presented at the 25th annual meeting of the Gesellschaft für Neuropsychologie, Madgeburg, Germany.

15. Forkmann $T$, Boecker $M$, Wirtz $M$, Glaesmer $H$, Brähler $E$, et al. (2010) Validation of the Rasch-based depression screening in a large-scale German general population sample. Health Qual Life Outcomes 8: 105.

16. Powers WJ, Derdeyn CP, Biller J, Coffey CS, Hoh BL, et al. (2015) American Heart Association Stroke Council. Guidelines for the early management of patients with acute ischemic stroke regarding endovascular treatment: A guideline for healthcare professionals from the American Heart Association. Stroke 10: 3020-3035.

17. Hemphill JC 3rd, Greenberg SM, Anderson CS, Becker K, Bendok BR, et al. (2015) Guidelines for the management of spontaneous intracerebral hemorrhage: a guideline for healthcare professionals from the American heart association/American stroke association. Stroke 7: 2032-2060.

18. Sacco RL, Kasner SE, Broderick JP, Caplan LR, Connors JJ, et al. (2013) An updated definition of stroke for the 21st century: a statement for healthcare professionals from the American Heart Association/American Stroke Association. Stroke 44: 2064-2089.

19. Pisters R, Lane DA, Nieuwlaat $R$, de Vos CB, Crijns $H J$, et al. (2010) A novel user-friendly score (HAS-BLED) to assess 1-year risk of major bleeding in patients with atrial fibrillation: The Euro Heart Survey. Chest 138: 1093-1100.

20. Nasreddine ZS, Phillips NA, Bédirian V, Charbonneau S, Whitehead V, et al. (2005) The Montreal Cognitive Assessment, MoCA: a brief screening tool for mild cognitive impairment. J Am Geriatr Soc 53: 695-699.

21. Royall DR, Cordes JA, Polk M (1998) CLOX: an executive clock drawing task. J Neurol Neurosurg Psychiatry 64: 588-594.

22. Morris JC, Heyman A, Mohs RC, Hughes JP, van Belle G, et al. (1989). The Consortium to Establish a Registry for Alzheimer's Disease (CERAD). Part I. Clinical and neuropsychological assessment of Alzheimer's disease. Neurology 39: 1159-1165.

23. Schmid NS, Ehrensperger MM, Berres M, Beck IR, Monsch AU (2014) The Extension of the German CERAD Neuropsychological Assessment Battery with Tests Assessing Subcortical, Executive and Frontal Functions Improves Accuracy in Dementia Diagnosis. Dement Geriatr Cogn Dis Extra 4: 322-334.

24. Reitan $R$ (1979) Trail-making test. Arizona: Reitan Neuropsychology Laboratory.

25. Rasmussen LS, Larsen $K$, Houx $P$, Skovgaard $L T$, Hanning CD, et al. (2001) ISPOCD group. The International Study of Postoperative Cognitive Dysfunction. The assessment of postoperative cognitive function. Acta Anaesthesiol Scand 3: 275-289.

26. Herm J, Fiebach JB, Koch L (2013) Neuropsychological effects of MRI-detected brain lesions after left atrial catheter ablation for atrial fibrillation: long-term results of the MACPAF study. Circ Arrhythm Electrophysiol 6: 843-850.

27. Kochhäuser S, Lohmann HH, Ritter MA, Leitz $P$, Güner $F$, et al. (2015) Neuropsychological impact of cerebral microemboli in ablation of atrial fibrillation. Clin Res Cardiol 3: 234-240.

28. Fanning JP, Walters DL, Platts DG, Fraser JF (2014) Characterization of neurological injury in transcatheter aortic valve implantation: how clear is the picture? Circulation 4: 504-515. 
29. Bendszus M, Stoll G (2006) Silent cerebral ischaemia: hidden fingerprints of invasive medical procedures. Lancet Neurol 5 : 364-372.

30. Mathews Abner E, Caban-Holt A, Kryscio R, Schmitt F (2013) CERAD practice effects and attrition bias in a dementia prevention trial. Int Psychogeriatr 7: 1115-1123.
31. Buck KK, Atkinson TM, Ryan JP (2008) Evidence of practice effects in variants of the trail making test during serial assessment J Clin Exp Neuropsychol 30: 312-318. 